\title{
Wie übergeht oder ignoriert man eschatologisches Gedankengut? Und warum? \\ Und wenn man es einmal übergangen oder verkannt hat, was wären dann konkret die Folgen?
}

\author{
Andrew Gow
}

Das programmatische Exposé der Organisatoren beantwortete den ersten Teil dieser Fragen prägnant und präzise: „Allerdings liegt der hochsäkularisierten europäischen Gesellschaft diese Art religiös motivierten Planens und Handelns fern und ist der europäischen politischen Kultur so fremd geworden, daß sie sie gar nicht erkennt, nicht als wirkmächtig ernst nimmt, oder aber ihre Wirkmächtigkeit nicht einschätzen kann." Nicht nur der europäischen politischen Kultur, sondern auch der rationalen akademischen und verwaltungstechnischen Kultur und Denkweise gebildeter Abendländler insgesamt ist diese Sicht und dieser Handlungsgrund fremd geworden und geblieben. Ich will hier keine weiterführende Kritik an der etwas eurozentrischen Färbung in diesem Passus des Exposés aufnehmen, aber die Ordnungsgröße scheint mir in diesem Falle eine Nummer zu klein geraten zu sein. Weiter ist der (implizite) Amerikabegriff, als mitgedachter Gegenpol zum Europabegriff, etwas zu schroff gegensätzlich formuliert: Das Eindringen evangelikaler Elemente eschatologischer Färbung in die US-amerikanische politische Klasse und Führungselite ist relativ neueren Datums (erst in den Reagan-Jahren, danach allerdings nur in der Epoche von Bush filius); und es leben ja noch außerhalb der Vereinigten Staaten und der EU weitere hunderte von Millionen Menschen, von denen man sagen kann, sie hätten praktisch als ,dritte Größe' einen bedeutenden Anteil an den politischen, kulturellen und intellektuellen Traditionen des Abendlandes - welche sie genauso schlecht auf das Verständnis ,unserer' eschatologischen Phänomene vorbereiten. Historiker, Sozialwissenschaftler, Politologen, Journalisten, ja auch Politiker, Regierungsbeamte und Sicherheitsbeamte aller, westlichen' Länder: alle (mit der möglichen Ausnahme, rechter ' evangelikaler Kräfte in den USA) stehen solchen Motivationen und Handlungsgründen noch ziemlich ratlos gegenüber, scheint es sich doch dabei um eine Wiederkehr des Verdrängten zu handeln, um längst widerlegte Mären und Altweibergeschichten einer früheren, schon überwundenen Kulturstufe... Und da begehen die Modernen ihren klassischen, trotz aller Säkularisierung doch religiös verbrämten Fehler - sie nehmen ihre eigene Epoche auch für das Ende, für das nec plus ultra bzw. für die 
(zeitweilige) Vervollkommnung menschlicher Entwicklung - selbst eine seltsam ironische Fehleinschätzung eschatologischer Prägung!

Wenden wir uns nun den Kollegen zu: Vor allem sind es Historiker, die eschatologisches Gedankengut in mittelalterlichen Prachtbibeln, in eschatologisch geladenen Texten und Zeugnissen der Besorgnis um die vorletzte Jahrtausendwende oder in den vielen Legenden und Geschichten über den vermeintlichen ,Antichrist ${ }^{\prime}$ nicht wahr- und ernstnehmen wollen; und obwohl die heutige evangelikale Apokalyptik und fundamentalistische muslimische Weltendeszenarien nun endlich doch ernst genommen werden - in manchen Kreisen -, pflegen sie doch in der Öffentlichkeit nur als extravagante Ketzereien gegen den rationalen Geist des Zeitalters und nicht als wirkmächtige Alternativen zu diesem sonst triumphierenden Geiste zu erscheinen.

Wie ich schon 1995 in meinem Buch „The Red Jews" ${ }^{1}$ dargelegt habe, ist die Figur des Antichrist schon seit der Spätantike Bestandteil einer durch und durch judenfeindlichen Enzeitschau. ${ }^{2}$ Danach seien die Juden in Anlehnung an die relevanten Stellen in den christlichen Schriften nicht durch Zwang zu bekehren, denn sie seien bis ans Ende der Zeit Zeugen für die Verwerfung Israels und des neuen, geistlichen Bundes mit den Christen; am Ende der Zeit werde danach noch ein Splitter der verbliebenen Juden zum wahren christlichen Glauben bekehrt, und die Prophezeiungen über den „dürren Baum“ und den aufgepropften Ast werden sich bewahrheiten. Dies ist verschiedentlich „die beste Variante des christlichen Antijudaismus" genannt worden, doch wird man nicht verkennen, daß Juden und Judentum darin eine ontologisch völlig untergeordnete und zugleich eine hochwichtige eschatologische Rolle spielen: als notwendige Statisten im letzten Drama, in dem Christus, Teufel, Gottvater und christliche Seelen die prominenten Hauptrollen besetzen. Dabei ist zu bedenken, daß die wörtliche und traditionelle Auslegung der relevanten Stellen in der Offenbarung des Johannes die Mehrheit der verbliebenen Juden zu Anhängern des falschen Messias, also des Antichrist, erklärt, und im blutgetränkten Endkampf bei Megiddo (,Armageddon') ermordet werden läßt.

Die antike Tradition, die in der tiburtinischen Sibylle einen prägnanten Ausdruck fand, war ein wichtiger, doch bislang wenig beachteter Bestandteil mittelalterlicher Endzeitvorstellungen. Hrabanus Maurus (ca. 776-856), Erzbischof von Mainz, brachte diese Vorstellung im 9. Jahrhundert auf den Punkt: „Die Juden träumen vom Kommen ihres Christus, der, wie wir wissen, der

1 The Red Jews. Antisemitism in an Apocalyptic Age, 1200-1600 (Leiden: E.J. Brill, 1995).

2 Siehe auch Andrew Gow, „The Jewish Antichrist in Medieval and Early Modern Germany“, in: Medieval Encounters 2,3 (1996), 249-285. 
Antichrist ist. ${ }^{\text {"3 }}$ Ein Reim des neunten Jahrhunderts vermittelt kompakt und detailreich den allgemeinen Glauben:

Antichristus est venturus permitente domino

In Babilonia nascetur conceptus de diabolo

Dan de tribu erit ortus ex Ebreorum populo. ${ }^{4}$

Die Epistola de antichristo des Abtes Adso de Montier-en-Der (910-992), um 950 geschrieben, ist eine Kompilation verschiedener frühmittelalterlicher $\mathrm{Zu}$ sammenfassungen patristischer Lehren über den Antichrist. ${ }^{5}$ Dieser Brief nimmt die Form einer typischen Heiligenvita an. Im Gegensatz zur tyconischen Tradition, nach der der Antichrist als eine Körperschaft aller Feinde der Kirche konzipiert wird, ${ }^{6}$ eine Denkrichtung, die stark aus Hieronymus und Augustinus gespeist wurde, geht es im Briefe des Adso um die persönliche und historische Gestalt, nicht um das Kollektive oder das Moralische.

In direkter Anlehung an die Auslegung des 2. Thessalonicherbriefes 2, 4 durch Heimo von Auxerre, ${ }^{8}$ welche Adso fast wortwörtlich übernahm, be-

3 Friedrich Murawski, Die Juden bei den Kirchenvätern und Scholastikern (Berlin, 1925), 36.

4 Richard Emmerson, Antichrist in the Middle Ages. A Study of Medieval Apocalypticism, Art and Literature (Seattle: University of Washington Press, 1981), 83. Siehe MGH. Poet. IV/2: Poetae Latini aevi Carolini, rec. K. Strecker. Berlin 1923, 644 (no. 88,2).

5 Zum Brief Adsos vgl. Horst-Dieter Rauh, Das Bild des Antichrist: von Tyconius zum deutschen Symbolismus (Münster, 1973), 153-164 und insbesondere das Standardwerk über Adso: Robert Konrad, De ortu et tempore Antichristi. Antichristvorstellung und Geschichtsbild des Abtes Adso von Montier-en-Der (Kallmünz, 1964). S.a. Bernard McGinn, Visions of the End. Apocalyptic Traditions in the Middle Ages (New York: Columbia University Press, 1979), 82-83.

6 D.h., Ketzer oder Juden, ja sogar der Teufel selbst (das war eine geläufige Deutung in der Zeit 500 bis 900); siehe Rauh, Das Bild des Antichrist im Mittelalter, passim.

7 Die exegetische Methode des Tyconius (4. Jhdt.) lag der spirituellen - im Gegensatz zur ,fleischlichen' (d.h., literalen) - Schriftauslegung zugrunde, insbes. der hebräischen Schriften (in denen T. auch die undurchsichtigsten Stellen als figurae der Kirche deutete); siehe Rauh, Das Bild des Antichrist, 102-121.

8 Haimo wurde lange Zeit fälschlich mit Haimo, Benediktiner und Bischof von Halberstadt $(\dagger 853)$ verwechselt oder gar identifiziert. Der Auxerrois war Benediktinermönch im Haus Saint-Germain-d'Auxerre und starb um 875. Sein Kommentar zur Offenbarung („Expositio in Apocalypsim“, PL CXVII, 937-1220) stand unter dem Einfluß des Ambrosius Autpertus (†778); die Glossa ordinaria schöpfte ihrerseits aus Haimo. Siehe auch Henri Barré, „Haymon d'Auxerre“, in: Dictionnaire de Spiritualité ascétique et mystique, Bd. VII (Paris: Beauchesne, 1969), Teil I, Sp. 91-97; Kevin L. Hughes, "Haimo of Auxerre and the Fruition of Carolingian Hermeneutics“, in: Second Thessalonians: Two Early Medieval Apocalyptic Commentaries: Haimo of Auxerre, Expositio in Epistolam II ad Thessalonicenses and Thietland of Einsiedeln, In Epistolam II ad Thessalonicenses (trans. Steven R. Cartwright and Kevin L. Hughes; TEAMS Commentary Series; Kalamazoo, MI: Medieval Institute Publications, 2001); und Deborah 
hauptete der Abt, der Antichrist sei weder Heide noch Christ, sondern ein Jude - und nicht nur irgendeiner, sondern der falsche Messias. Er beschneidet sich selbst und behauptet, er sei der ,Christus', also der Gesalbte, der Messias, der den Juden verheißen wurde; diese, im Glauben ihren Messias zu empfangen, empfangen an seiner Stelle den Teufel.?

Obwohl Adso die tiburtinische Sibylle nicht kannte, kannte er doch die dem Methodius zugeschriebenen „Revelationes“, in denen der Antichrist als Individuum dargestellt wurde. Adso hat diesen Mythos für den lokalen, westfränkischen Gebrauch ,verwestlicht' und den rex Francorum, seinen Mäzen, anstelle des ,Königs der Römer' oder des ,Kaisers der Römer und Griechen' eingesetzt. Diese Elemente der Legende in der Version Adsos, die vielfach wiederholt und von einer reichen handschriftlichen Tradition verbreitet wurde, haben spätere Ideen über den Antichrist im Westen maßgeblich beeinflußt. ${ }^{10}$

Bernard McGinn ist Professor für Religious Studies an der renommierten Divinity School der University of Chicago. Ausgetretener Priester der katholischen Kirche und Professor der Religionswissenschaft (und nicht etwa der Theologie), ist er sowohl ,kirchennah' wie ,kollegennah', was die Chicago School der kritischen Religionswissenschaft angeht. Diese Richtung läßt es nicht an Erkenntnismustern und Anregungen aus anderen Disziplinen fehlen - ja, im Gegenteil, sie lebt gerade von diesem oft fruchtbaren Zwiegespräch. Sein bestbekanntes Buch heißt „Antichrist. Two Thousand Years of Human Fascination with Evil“. Allein der Titel läßt erkennen, worum es dem Autor in diesem Buch von 1994 geht. McGinns psychologisierende Analyse schreibt der Antichristfigur eine permanente, sich durch die Zeiten nicht wandelnde Dynamik der Projektion zu: „Antichrist“ sei immer und überall im christlichen Abendland ein Blitzableiter für allerlei Angst vor dem Bösen. Daß die Figur des Antichrist in verschiedenen historischen Lagen, etwa in der Reformationszeit, auch dazu gedient hat, das Böse stellvertretend und biblisch autorisiert zu vertreten, wird jeder zugeben; daß dies die primäre Rolle des im Mittelalter sehr populären spätantiken Konglomerats aus verschiedensten Bibelstellen, sibylli-

Everhart, Commentary on the Book of Jonah: Haimo of Auxerre (trans. Deborah Everhart; TEAMS Commentary Series; Kalamazoo, MI: Medieval Institute Publications, 1993).

9 Rauh, Das Bild des Antichrist, 161; Sackurs Edition des „Libellus de ortu et de tempore Antichristi“, 111; PL CI, 1295d - 1296a; cf. Haimo, PL CXVII, 779d - 780b.

10 Im 11. und 12. Jhdt. erschienen mindestens sieben verschiedene lateinische Fassungen von Adsos Epistola: McGinn, Visions of the End, 84. Eine Edition des Brieftextes stammt von Ernst Sackur: „Epistola Adsonis ad Gerbergam reginam de ortu et tempore Antichristi“, in: Sibyllinische Texte und Forschungen: Pseudomethodius, Adso und die tiburtinische Sibylle (Halle, 1898), 97-113, sowie eine neuere: Daniel Verhelst, „Adso Dervensis: De Ortu et Tempore Antichristi“, in: Corpus Christianorum, Continuatio Medievalis XLV. Siehe McGinn, Visions of the End, 84-87, für die betreffenden Stellen in seiner Einführung. 
nischen Weissagungen, der Alexanderlegende und anderen weiteren Legenden gewesen sein kann, wird man genauso entschieden bestreiten müssen.

Die genauen Schriftpassagen, die die Kernbestandteile dieser apokalyptischen Mélange ausmachten, sind nicht alle eschatologischer Art - etwa die Timothäus-Stellen - doch die Identifikation der Bestie in der Offenbarung mit dem Antichrist läßt keinen Zweifel zu: Es handelt sich primär um eine eschatologische Figur, die auch je nach Sachlage zu anderen Zwecken gebraucht werden konnte: ein Papst, ein Kaiser, ein Erzketzer oder ein Gegner jeglicher Art (Tartar, Türke, Unruhestifter) konnte immer wirksam als Antichrist beschimpft werden, egal ob dadurch eine Rolle im Endzeitdrama anvisiert wurde oder nicht. Die Vielfalt dieser Möglichkeiten liest man an der großen Arbeit des Horst-Dieter Rauh ab, dessen Werk über den Antichrist von Tyconius bis ins hohe Mittelalter den ekklesiologischen und politischen Gebrauch der vielumstrittenen Figur ausführlich darlegt. Wie kann man McGinns außerordentlich gute Kenntnisse der Quellen mit seiner seltsam anämischen und anodynen Auslegung derselben in Übereinstimmung bringen?

Parallel zu seinem bewußten Herunterspielen der eschatologischen Rolle des Antichrist versucht McGinn, der Literatur zum Antichrist ihre oft rohe Judenfeindlichkeit oder, präziser ausgedrückt, den Stachel ihres Antijudaismus zu nehmen. Daß der Antichrist in den meisten nicht-politischen Rollen des Mittelalters als Jude erscheint, wird weder von McGinn noch von den meisten anderen Fachleuten wahrgenommen; daß das Judentum des Antichrist dann mit zentralen Elementen der christlichen Eschatologie, sowohl auf gelehrter wie auch auf der Volksebene, zusammenhing, wird von ihm und in der Mehrheit der wissenschaftlichen Literatur mehr oder weniger totgeschwiegen - als ob es gelte, eine nachweisbar offiziell gebilligte christliche Tradition von einem solchen Schandflecken zu reinigen. Übergeht oder übersieht er das eschatologische Moment an der Antichristlegende? Ja, und zwar aus apologetischen Gründen, die seine ganze große und ausführliche Arbeit letztendlich zum Blindgänger machten.

Sara Lipton hat eine vollständige kunstgeschichtliche Analyse der Bibles moralisées des frühen 13. Jhdts. geliefert. ${ }^{11}$ Dabei ging sie erstens von formalistischen kunsttheoretischen Voraussetzungen aus, deren Wert insgesamt hier nicht angefochten werden soll, nur insofern hinterfragt, als daß es sich in den Pariser Bibles moralisées nicht ausschließlich um ein Werk kirchlicher oder monastisch-gelehrter Prägung handelt, sondern um ein Werk, das eben in der Volkssprache das Geschehen und die Moralia der Bibel für ein wohl königliches, aber des Lateinischen unkundiges, also ,illiterates' Publikum darstellen sollte.

11 Sarah Lipton, Images of Intolerance. The Representation of Jews and Judaism in the Bible moralisée (Berkeley/Los Angeles/London: University of California Press, 1999), und siehe meine Rezension in Speculum 76,3 (July 2001), 756-758. 
Das Bildprogramm stellt in den Vordergrund eben auch exegetische Traditionen, die in der gelehrten Theologie und im Schriftstudium relativ wenig Platz beanspruchten, und zwar die schon erwähnte eschatologische Fixierung auf die Rolle der Juden in den letzten Tagen. Lipton geht den konkreten, bildlichen, kulturellen und mäzenatischen Umständen der Entstehung dieses Werks eingehend nach, nur verpaßt sie den Zugang zur hochgradigen Apokalyptik der durchaus judenfeindlichen und antijudaistischen Illustrationen von Juden als Vasallen des Antichrist - weil sie die betreffenden Traditionen einfach nicht wahrnimmt. Ihre Ausbildung in den bis vor kurzem fast ausschließlich der mittelalterlichen Hochkultur zugewandten graduate programs in Geschichte in Harvard und die weitere Betreuung des Projekts durch Robert Chazan, den amerikanischen "Chef" der Geschichte der Juden im Mittelalter, bietet den Kontext ihrer Auslassung: Sie wird wohl wenig oder nichts von eschatologischen Traditionen im Bereich der sogenannten ,vernacular theology' gewußt haben. Diese Ideen sind trotz der abweisenden Haltung der Schultheologen sowohl im Frühmittelalter wie auch im 15. Jahrhundert überall zu verspüren: doch außerhalb des theologischen und literarischen Kanons. Auch die Lektüre von „The Red Jews" scheint bei Sara Lipton nichts gefruchtet zu haben, übergeht sie doch fast stillschweigend die markanten Parallelen zwischen den Abbildungen von Juden um den Antichrist herum in ihren Manuskripten und den von mir kommentierten Beschreibungen von der Rolle der Juden als Vasallen des Antichrist in den letzten Tagen aus früheren und gleichzeitigen Texten. Das heißt konkret: Die wiederholte, explizite und deutlich judenfeindliche Darstellung von Juden als Vasallen des Antichrist, die scharf konturiert in den Bibles moralisées begegnet, bleibt ihr unverständlich und folglich verschlossen. Somit wird die eingangs zitierte Einschätzung abendländischer Blindheit für eschatologische Momente in Vergangenheit und Gegenwart seitens der Organisatoren zum Paradigma für unser Verständnis einer leider verfehlten wissenschaftlichen Literatur. Ein weiteres Beispiel wird dies wohl ausreichend belegen.

Sylvain Gouguenheim bietet in seinem Buch „Les fasses terreurs de l'an mil: attente de la fin des temps ou approfondissement de la foi ?"12 (was ich als ,Die vermeintlichen Schrecken des Jahres 1000' übersetzen würde) allen, die in der engeren Zeit vor der Jahrtausendwende apokalyptische Spekulation, Zeitrechnungen, Naherwartung oder date fixing, also eschatologische Wettervoraussagen zu finden meinen, eine dezidiert ideologisch getränkte Kritik ihrer freilich genauso ideologisch geprägten Geschichtsschreibung. Er behauptet, in den Quellen des 9. und 10. Jahrhunderts so gut wie keine eschatologische Naherwartung zu finden: Doch scheint er nur die Theologen und die gebildeten

12 (Paris: Picard, 1999). Siehe auch Johannes Fried, „Die Endzeit fest im Griff des Positivismus? Zur Auseinadersetzung mit Sylvain Gouguenheim“, in: HZ 275 (2002) 281 321, der Gouguenheim umfassend widerlegt. 
Schriftexegeten ernst zu nehmen. Es kann sehr wohl der Fall gewesen sein - war in der Tat der Fall für die meisten gebildeten Kleriker -, daß die offizielle Kirche die skeptische Stellung des Augustinus und des Hieronymus streng eingehalten hat: mit Verweis auf Matthäus 24, 42 hatten beide davor gewarnt, allzu deutlich $\mathrm{zu}$ wissen zu versuchen, wann und wie das Ende anbrechen sollte. Doch andere Zeugen, nicht wenige, wie Johannes Fried schon auf deutsch und auf englisch mit Bezug auf den ottonischen Kaiserhof gezeigt hat, deuten auf eine weitverbreitete Naherwartung des Endes um das Jahr 1000 herum. ${ }^{13}$ Der teilweise in Paris erzogene Jude Richard Landes, vom überdeutlich französichen Gougenheim mit einer Geste unverfrorener Kulturarroganz immer wieder als „l'américain" apostrophiert, plädiert seit mehr als einem Jahrzehnt für das Ernstnehmen aller möglichen Quellen, die uns erlauben könnten, die eschatologische Temperatur des Patienten ,Lateinisches Christentum' um das Jahr $1000 \mathrm{zu}$ messen. Dabei überspringt er, teilweise durch sehr genaue Kontextkenntisse beflügelt, teilweise durch die ihm eigene strukturelle Fantasie, die Aussagen der normativen Quellen und verspürt, mit einer Mischung aus Quellenakribie und Empathie für eschatologische Denkarten, sowohl ein sich steigerndes Moment apokalyptischer Naherwartung vor dem Jahr 1000 - er sieht etwa die Treuga Dei und verschiedene Reformbewegungen als Folgen dieser Erwartung - wie auch wiederholte Episoden apokalyptischer Erwartung und verwandten Handelns in den Jahrzehten direkt nach 1000, also gelegentlich der ersten großflächigen Ausschreitungen gegen Juden im Abendland um 1009-1010 herum ${ }^{14}$, nachdem der Kalif Al-Hakim die Zerstörung der Kirche des Heiligen Grabes angezettelt hatte, und die Jerusalemer Judenschaft dafür mitverantwortlich gemacht wurde (jedenfalls im Okzident). Mit ein Grund für diese Ausschreitungen wäre also der Glaube gewesen, in Anlehnung an die Ansichten etwa eines Adso, daß Juden eben als Diener des Antichrist ihm schon den Weg bahnen würden (wie eine apokalyptische ,fünfte Kolonne'); im Bau der vielen neuen Kirchen - auch von Radulfus Glaber bezeugt -; und im Vorfeld zum Passionsjahr 1033.

13 Johannes Fried, „Endzeiterwartungen um die Jahrtausendwende“, in: DA 45, 2 (1989), 385-473; eine englische Fassung, leider ohne wissenschaftlichen Apparat, erschien als „Awaiting the End of Time Around the Turn of the Year 1000", in: Richard Landes, Andrew Gow und David C. Van Meter, Hrsg., The Apocalyptic Year 1000. Religious Expectation and Social Change, 950-1050 (Oxford/New York: Oxford University Press, 2003), 17-63.

14 Richard Landes, „The Massacres of 1010: On the Origins of Popular Anti-Jewish Violence in Western Europe", in: From Witness to Witchcraft: Jews and Judaism in Medieval Christian Thought, hrsg. von Jeremy Cohen (Wolfenbüttel, 1996), 79-112; zwischen 1012 und 1014 dienten verschiedene Wunder und Naturkatastrophen als Vorwand für die Vertreibung der Mainzer Judenschaft und schürten bei manchen den Verdacht, die Welt kehre in ihren ursprünglichen Zustand des Chaos zurück: Annales Quedlinburgenses, $M G H$, SS 3, 81-83. 
Gougenheim hat insofern recht, daß man nur aufgrund von Auslassungen, Schweigen und Lücken nicht direkt auf Geschichtsfälschung, Zensur oder gar Verschweigen der Tatsachen folgern kann; doch darf man annehmen (wenn nicht mit der gleichen Inbrunst eines Gouguenheim), daß viele gebildete Kleriker ihren Augustinus kannten, sich und ihre Zeitgenossen nicht der Nachwelt lächerlich machen wollten und dementsprechend die Quellen zurechtschrieben. Und im Lichte der spärlichen Quellen, die auf verschiedene Episoden apokalyptischer Naherwartung oder gar Panik deuten, scheint die Möglichkeit einer gewissen Selbstzensur nicht so abwegig. Will man nur die Standardquellen der Ideen-, Rechts- und Institutionsgeschichte gelten lassen, und verschreibt man sich einer positivistischen Modernität als Kriterium nicht nur fürs Studium fremder Gedankenwelten, sondern auch als vermeintliche Richtlinie für die Bewertung und Extrapolation solcher Gedankenwelten, so wird man die Weisungen der abendländischen Kirchenväter, die zwar in den gelehrten, also normativen Quellen wiederholt werden, nicht nur als Richtlinien sehen, sondern als Wahrheitsbeschreibung schlechthin, ${ }^{15}$ und diese dann eisegetisch in alle anderen Quellen, ob Kaiserchronik oder Radulfus Glaber, einlesen wollen. D.h., man kann ziemlich einfach die hortative Funktion solcher Texte vergessen, dienten und dienen sie heute noch unter Christen zur Mahnung gegen Versuche der apokalyptischen Wettervoraussage. Man wiederholt ja eine Mahnung erst dann, wenn sie vonnöten ist. Wollte man vor dem Jahr 2000 keine Angst schüren, wollte man sogar im Geiste des säkularisiertesten aller westlichen Staaten jeden Millennium-Rummel im voraus für abergläubigen Unfug erklären, so hatte man auch guten Grund, in der Vergangenheit keine irrationalen Ausschweifungen dieser Art finden zu wollen.

Doch sind es nicht nur Wissenschaftler, die imstande sind, eschatologisches Gedankengut schlichtweg zu ignorieren oder zu verkennen. Das geschieht auch in ähnlicher Weise in der Öffentlichkeit, im politischen und religiösen Bereich.

\section{Waco, David Koresh}

Das FBI konnte nicht erkennen, daß es Koresh um das Ende der Welt ging Koresh sah für die Bundespolizeibehörde aus wie ein für dortige Verhältnisse gewöhnlicher schwerbewaffneter Spinner und Sektenführer. Statt Experten mit

15 So z. B. Sylvain Gouguenheim, der in seinem zweiten Kapitel (Les fausses terreurs, 6592) seinen Glauben kundtut, die augustinische Theologie habe das ganze Zeitalter derart dominiert, daß auch der explizite Verweis des Predigers Radulfus Glaber auf das Jahr 1000 als das Jahr der Befreiung des Antichrist nicht als apokalyptisch gelten darf, weil Glaber ,diesen exegetischen Rahmen [von Augustin] nicht außer Acht hätte lassen können.' (170). 
dem relevanten Wissen um apokalyptische Vorstellungen und Glaubensmuster nach Waco zu holen, hat man einfach die Koresh-Leute unter polizeiliche Bewachung gestellt und schließlich attackiert, im vollen Vertrauen, es handele sich um einen gewöhnlichen Kampf des Willens und man könnte die Lage wie eine Kreuzberger Hausbesetzung mit schlichter Gewalt beenden. Das FBI überging schlicht das hochbrisante eschatologische Gedankengut von David Koresh und seinen Anhängern - und das kostete vielen Menschen das Leben. Seitdem hat das FBI ein gewisses Umdenken für nötig erachtet und sogar Fachleute herangezogen für das weitere Studium anderer eschatologischer Denkmuster.

Im Herbst 2005 entfachte ein sonst unbekannter Imam in Vancouver einen Mediensturm, indem er seiner Gemeinde predigte, am Ende der Zeit würden selbst die Steine und die Bäume die sich verbergenden Feinde des Islam anzeigen, daß man sie mit Steinen töte - doch ist dies eine Standardstelle, die nicht unbedingt einen antijüdischen Inhalt hat. Doch als er diese Stelle in Verbindung mit der bekannten Beschimpfung der Juden als Nachkommen von Affen und Schweinen in Verbindung brachte, hat dieser einfallsreiche Imam nun ein wahres antijüdisches Gift gemischt - was aber in den Medien aufgefallen ist, war der vermeintliche Judenhaß, nicht der eschatologische Inhalt seiner Zitate aus dem Koran. ${ }^{16}$ Was uns beschäftigen sollte, ist weder das eine noch das andere, sondern die fatale Mischung dieser Elemente, die der Religionswissenschaftler allzu gut erkennt - und zwar aus Geschichte und Gegenwart des Christentums.

\section{Die spekulative „Fiktionsreihe“ Left Behind}

Im europäischen Kontext fast undenkbar ist der enorme Erfolg einer Reihe spekulativtheologischer Action-Romane von den vor-millennialen Dispensationalisten (pre-millennial dispensationalists) Tim LaHaye und Jerry B. Jenkins. Diese Reihe von Zukunftsromanen über die kommende Endzeit, ;Left Behind', also ,Hinterlassen', hat unglaubliche Auflagen in den Vereinigten Staaten, mit einem Verkaufsvolumen von insgesamt 50 Millionen Bänden. Wissenschaftler aller Couleur haben gezögert, sowohl den wachsenden Einfluß in der Öffentlichkeit fundamentalistischer Diskurse wie auch dieses publizistische Phänomen ernst zu nehmen - teilweise wohl weil Wissenschaftler, die sich mit Populärreligion befassen, selten die sehr einfachen Ideen über die göttliche Urheberschaft oder die unzweideutige Auslegung der Heiligen Schrift des ,religiösen Rechten' teilen. Man sieht weg und hofft, den Leuten nicht mehr Aufmerksamkeit zu verschaffen, als sie schon bekommen. Für dieses Wegsehen ist die

16 Siehe die Meldung im staatlichen kanadischen Nachrichtendienst CBC vom 22.10.04: http://www.cbc.ca/story/canada/national/2004/10/21/abubaker041021.html, Stand des 22.03.06. 
Zeit nun vorbei, erreichte doch das gruselige Machwerk Desecration für das Jahr 2001 die erste Stelle für ,hardcover fiction' (also für neue Titel) in der Bestsellerliste der Zeitschrift „Publishers Weekly“. ${ }^{17}$

In der Zeitschrift „U.S. News \& World Report“ verdeutlichte Jeffrey L. Sheller das evangelikale Programm hinter dieser Reihe:

...many evangelicals view the 1948 founding of Israel as the first in a chain of biblically mandated events that will culminate in the return of Jesus and the end of history-a scenario popularized in the bestselling Left Behind fiction series. Part of that scenario, based on a hotly debated interpretation of passages in Revelation, Daniel, and Ezekiel, involves a devastating 'battle of Armageddon'- a nuclear holocaust that is stopped at its climax by the arrival of Jesus, who defeats the forces of evil and establishes an era of peace. But millions of Jews are killed in the battle, and the survivors accept Jesus as their Messiah. ${ }^{18}$

Donald Rumsfeld und George W. Bush, um nur zwei führende Figuren zu nennen, stehen dem betreffenden Strang des von der zunehmend rechten Southern Baptist Church beherrschten evangelikalen Christentum nahe; Rumsfeld selbst hält dieses Szenario für gewiß, doch der amerikanische Präsident, ein born-again Christian (,wiedergeborener Christ'), hat sich noch nicht direkt dazu geäußert. Woher kamen diese Ideen ins evanglikale Lager? Wir haben schon ein wenig den spätantiken Hintergrund dieser wohl halb-biblischen, halb-literarischen Ideen über den Antichrist eruiert. Trotz der Ablehnung fast aller Schulmänner und der Reformatoren lebten diese Ideen auf einer anderen Stufe der Bibelauslegung und des Bibelverständnisses weiter und wurden wohl von den Anhängern des Gründers des ,pre-millennial dispensationalism', John Darby, im 19. Jhdt. aus puritanischen und sonstigen Texten aus der Welt der englischen Dissenters aufgelesen und neu eingesetzt. Man begann, Ausschau zu halten nach Zeichen des Antichrist. Die Staatsgründung Israels 1948 legte zugleich den Grundstein für ein apokalyptisches Narrativ. Nicht nur evangelikale Christen, sondern auch Muslime, etwa der Großmufti von Jerusalem, dachten und denken apokalyptisch über die Juden im Kontext Israels: Hadj Amin Al-Husseini sagte schon 1937 vor der Peel Commission, die ,Endabsicht der Juden' sei der Neuaufbau des Tempels auf dem Tempelberg. ,Endabsicht vielleicht - aber eben am Ende der Zeiten und nicht übermorgen! Neuere Versuche auf palästinensischer Seite, jeden jüdischen Bezug zum Haram alScharif/Har ha-Bayit zu leugnen, rühren wohl nicht von Immoblienwerten her, sondern von einem ängstlichen, etwas konfusen Verständnis der jüdischen Eschatologie und, wie ich meine, nicht ohne Widerhall so mancher christlicher apokalyptischer Szenarien. Daß die überwältigende Mehrheit aller jüdischen

17 http://www.leftbehind.com/article.asp?loc_id=1\&item_id=12636, Stand des 17.10.02.

18 August 12, 2002, pp. 34-35. 
Tradition vorschreibt, der Tempelbau könne nie durch Menschenhand, sondern allein durch den Messias geschehen, sei hier nur am Rande angemerkt.

Und hier wird es brenzlig: In unzähligen Texten legendären und ,subtheologischen' Charakters - was man heute eher vernacular theology nennt -, meist in den Volkssprachen, tritt der von den Juden erwartete Messias als der von den Christen gefürchtete Antichrist auf. Wir haben es mit einem Analogieschluß zu tun: Wir, die Christen, erwarten und fürchten das Kommen des Antichrist; sie, die Juden, warten und hoffen auf ihren Messias - also werden diese Gestalten wohl eine und dieselbe sein! Hilfreich war hier die Stelle Mt. 24, 5: „Und viele werden kommen und sagen ,Ich bin der Messias", und viele werden von diesen in die Irre geführt". Es ist hier kein Platz, um diesen doppelten messianischen Komplex im Detail vorzunehmen, doch hat er eine sehr lange und reiche Geschichte, die nun von einem großen Teil der amerikanischen Evangelikalen weitergeführt wird: Jerry Falwell, Dick Van Impe, Donald Rumsfeld und vielen anderen. Laut ;Left Behind" wird die Mehrheit der Juden dem Antichrist dienen; nur ein Teil Israels wird dessen wahren, teuflischen Charakter bemerken und sich rechtzeitig zu ,Jesus' bekehren - und so soll sich die evangelikale Auslegung der Prophezeiung im Römerbrief 11 bewahrheiten (trotz entgegengesetzter Meinung im 26. Vers, wonach, ganz Israel' gerettet wird). Wir haben es hier mit einem deutlich religiös geprägten Judenhaß zu tun, in dem man Anti-Judaismus von Judenhaß gar nicht mehr trennen kann.

Was gibt es hier zu bemerken? Nicht bloß eschatologisches Gedankengut, obwohl dessen Anteil und Einfluß in großen Kreisen der amerikanischen Führungselite in der Öffentlichkeit kaum wahrgenommen, geschweige denn ernst genommen wird, sondern apokalyptische Naherwartung in einem unheiligen Bündnis mit einem integralen rassisch-religiösen Feindbild. Davon weiß man heute im jungen wie auch damals im untergangenen alten Europa ein paar Lieder zu singen. Obwohl das breite Spektrum evangelikaler Amerikaner sich überhaupt nicht einig ist über das Szenario der pre-millennials, hegen doch vermutlich mehrere Millionen US-Amerikaner - und es gibt diese Form des radikalen Protestantismus fast nirgends sonst auf der Welt - die Vorstellung eines nahenden eschatologischen Konflikts, in dem Juden entweder sich bekehren oder hingemetzelt werden müssen. Ich glaube, daß man hier Ausschau halten muß nach Parallelbeispielen aus der Vergangenheit. Ich behaupte, und es teilt diese Behauptung nur ein Teil des religionswissenschaftlichen Faches, daß Zeiten intensiver eschatologischer Spekulation und Erwartungen apokalyptischer Art oft ein Klima des Judenhasses, ja des radikalen Anti-Judaismus, ermöglicht haben; und schließlich manchmal sogar die schlimmsten Ausschreitungen gegen Juden - mitunter als Knechte und Diener des gefürchteten Antichrist - ausgelöst haben. Schließlich dürfte man vielleicht auch die endzeitliche Utopie des ,Tausendjährigen Reiches' unseligen Gedächtnisses wohl in diesen Traditionsstrang einreihen. 
Es ist zu bedenken, daß nach dem 11.9. 2001 („9/11“) in den USA die Angst vor weiteren Angriffen eines Feindes, der hauptsächlich nicht politisch, sondern religiös definiert wird, zu Haß und zu Gewalt gegen Muslime und Farbige geführt hat, sowohl im Inland wie auch im Ausland, und zwar explizit in der Form eines Kreuzzugs, der ausgerufen wurde gerade von einem evangelikalen Machthaber - also gibt es bereits einen gegenwärtigen Parallelfall oder Beispiele für die Ausführung von religiösen Angstvorstellungen im Bereich der Taten. Daß das realpolitische Moment in der US-amerikanischen Regierung bisher den illegalen amerikanischen "Islam-Krieg" nicht zum Versuch werden läßt, das in den USA schon auf sehr breiter Basis verbreitete apokalyptische Szenario der pre-millennials zu realisieren, ist ein glücklicher Umstand, aber keineswegs ein natürlicher, auf den man sich weiter unbekümmert verlassen sollte.

Also gilt es m. E. sowohl auf dem Gebiet der wissenschaftlichen wie auch auf dem der politischen Auseinandersetzung das hochriskante Gefahrenmoment ,eschatologisches Gedankengut', wo immer möglich, nicht zu übersehen, öffentlich zu machen und auf seine Gefahren hinzudeuten. 